\title{
DEVELOPMENT OF TOLERANCE TO THE PHYTOALEXIN 6-METHOXYMELLEIN IN MICROORGANISMS
}

\author{
FUMIYA KUROSAKI, IZUMI SAKURAI AND ARASUKE NISHI \\ Faculty of Pharmaceutical Sciences, Toyama Medical and Pharmaceutical \\ University, Sugitani, Toyama 930-01, Japan
}

(Received November 21, 1983)

\begin{abstract}
6-Methoxymellein $(6 \mathrm{MM})$, a phytoalexin of carrot, exerted a static effect on the growth of fungi, yeast and bacteria at low concentrations. It retarded the spore germination of Chaetomium globosum and extended the lag phase in the growth of Bullera alba, Candida albicans and Staphylococcus aureus. When $B$. alba and $C$. albicans were first treated with $6 \mathrm{MM}$ and then a second time their growth was not inhibited by the second dose, suggesting that they become tolerant to $6 \mathrm{MM}$ through some induced factor. By contrast, $S$. aureus appeared to tolerate $6 \mathrm{MM}$ in a constitutive manner, since the bacterial growth was inhibited by the second dose of $6 \mathrm{MM}$ as well as the first. In $S$. aureus culture, however, the duration of growth inhibition by $6 \mathrm{MM}$ was dependent on the inoculum size, and the inhibitory effect was negligible when the bacterial concentration in the culture was sufficiently high. Chaetomium globosum and $B$. alba incorporated appreciable amounts of $6 \mathrm{MM}$, but no metabolite of the phytoalexin appeared in the culture. The results suggest that $6 \mathrm{MM}$ tolerance occurred in these microorganisms independently of its degradation or transformation.
\end{abstract}

Many higher plants produce antimicrobial compounds when they are challenged by microorganisms. These compounds known as phytoalexins, are believed to be involved in the defense reactions of plants $(1,2)$. Phytoalexinmediated resistance to pathogens may be influenced by a number of factors which involve the biosynthesis and degradation of the phytoalexin by plant cells and aquisition of tolerance by microorganisms. Phytoalexin tolerance by microorganisms has been studied by many workers, but how pathogens tolerate phytoalexin is not fully understood. Some fungi and bacteria have the ability to detoxify phytoalexins by converting them to less inhibitory compounds (3-8). Cases have also been reported in which microorganisms become tolerant to phytoalexin by a non-degradative mechanism $(7,9-11)$. 
We recently reported the toxicity of a carrot (Daucus carota) phytoalexin, 6-methoxymellein (6MM), to some fungi, yeasts and bacteria, and showed that the phytoalexin has a broad antimicrobial spectrum (12). In this paper, we wish to report the effect of 6MM on the growth of a filamentous fungus (Chaetomium globosum), two yeasts (Bullera alba and Candida albicans) and a bacterium (Staphylococcus aureus) and also the tolerance of these microorganisms to the phytoalexin.

\section{MATERIALS AND METHODS}

Isolation of $6 \mathrm{MM}$. Crystalline $6 \mathrm{MM}$ was prepared from carrot root slices infected by $C$. globosum according to the method of KUROSAKI and NIHSI (12).

Microorganisms and cultivation. Chaetomium globosum and B. alba were obtained from Dr. K. TsubaKI, Institute of Biological Sciences, Tsukuba University. Candida albicans was obtained from Dr. K. TANAKA, Faculty of Medicine, Nagoya University. These fungi and yeasts were maintained on an yeast-malt agar medium (yeast extract $0.4 \%$, malt extract $1 \%$ and glucose $0.4 \%$ ). Staphylococcus aureus (ATCC 25923) was obtained from Dr. K. Konishi, Faculty of Medicine of this university, and maintained on a heart infusion agar (Difco). Spore suspension of $C$. globosum were prepared by adding distilled water to a 3 week-old culture and filtering through one layer of gauze to remove large mycelial fragments. The spores were collected by centrifugation at $1,000 \times g$ for $10 \mathrm{~min}$, and stored at $4^{\circ}$ until use. For the germination assay, the spores were resuspended in the yeast-malt liquid medium at a concentration of $2 \times 10^{5}$ spores $/ \mathrm{ml}$ and agitated on a reciprocal shaker (72 strokes/min) at $27^{\circ}$. Bullera alba and C. albicans, kept on agar slants, were transferred to the yeast-malt liquid medium and similarly shaken at $27^{\circ}$. Staphylococcus aureus was cultured at $37^{\circ}$ in the heart infusion liquid medium with shaking. Prior to the experiments, these yeasts and bacteria had been pre-cultured in the respective liquid media for a period in which they were subcultured at least 3 times.

Determination of microbial growth. Microorganisms were cultured in test tubes (14 mm diameter) containing $5 \mathrm{ml}$ of medium. For use in bioassay, the $6 \mathrm{MM}$ was dissolved in ethanol and added to the culture to give a final solvent concentration of $2 \%$. Spore germination of C. globosum was observed under a microscope. The growth of other microorgansims was followed by comparing the turbidity at $540 \mathrm{~nm}$ with that of an uninoculated medium.

Preparation of ${ }^{14} \mathrm{C}$-labelled $6 \mathrm{MM} .{ }^{14} \mathrm{C}$-Acetate was used as the radioactive precursor of $6 \mathrm{MM}$, since KUĆ (1) suggested that the acetate-malonate pathway is the most likely route for the biosynthesis of $6 \mathrm{MM}$. Carrot slices $(200 \mathrm{~g})$ were inoculated with a 2 day-old culture of $C$. globosum, and after $3 \mathrm{hr}, 300 \mu \mathrm{Ci}$ of $2{ }^{14} \mathrm{C}$-sodium acetate (specific activity, $55 \mu \mathrm{Ci} / \mu \mathrm{mol}$ ) in $40 \mathrm{ml}$ water was sprinkled over them. Another $300 \mu \mathrm{Ci}$ of ${ }^{14} \mathrm{C}$-acetate was added to the culture after $6 \mathrm{hr}$ 
incubation. Further incubation was carried out at $27^{\circ}$ for $24 \mathrm{hr}$, then the infected carrot slices were immersed in acetone. The extraction and purification procedures for radioactive $6 \mathrm{MM}$ were the same as for the unlabelled compound. The radioactivity of the product was measured by a liquid scintillation spectrometer using a commercial scintillation cocktail (Amersham ACS II). The amount of ${ }^{14} \mathrm{C}-6 \mathrm{MM}$ was determined by a thin layer chromatography (TLC) scanning method at $\lambda_{\mathrm{S}} 265 \mathrm{~nm}$ and $\lambda_{\mathrm{R}} 400 \mathrm{~nm}$, as reported previously (12), and the specific activity was adjusted to $185,000 \mathrm{dpm} / \mathrm{mg}$ by diluting the ${ }^{14} \mathrm{C}-6 \mathrm{MM}$ with unlabelled $6 \mathrm{MM}$.

Metabolism study. Loss of 6MM from culture media was measured by determining the radioactivity of ${ }^{14} \mathrm{C}-6 \mathrm{MM}$ added to the cultures. Bullera alba, $C$. albicans and $S$. aureus were inoculated into $1 \mathrm{ml}$ of the respective media which contained $0.2 \mathrm{mM}{ }^{14} \mathrm{C}-6 \mathrm{MM}$ for $B$. alba and $C$. albicans and $1.0 \mathrm{~mm}$ for $S$. aureus. A spore suspension $(1 \mathrm{ml})$ of $C$. globosum was also supplemented with $1.0 \mathrm{~mm}$ ${ }^{14} \mathrm{C}-6 \mathrm{MM}$. At various intervals, $100 \mu \mathrm{l}$-aliquots were removed from the cultures, and $6 \mathrm{MM}$ was extracted from the culture fluid by blending with $100 \mu \mathrm{l} n$-butanol for $15 \mathrm{sec}$. After standing for $1 \mathrm{~min}, 50 \mu \mathrm{l}$ of $n$-butanol extract was applied to a silica gel TLC plate (Merck, silica gel $60 \mathrm{~F}$ 254) and the plate was developed for $15 \mathrm{~cm}$ in benzene-methanol $(100: 2, \mathrm{v} / \mathrm{v})$. The region containing $6 \mathrm{MM}$ was detected under an ultraviolet lamp, and scraped off. ${ }^{14} \mathrm{C}-6 \mathrm{MM}$ was extracted overnight from silica gel with $0.5 \mathrm{ml}$ ethanol and the radioactivity of the ethanol extract was measured.

A possible metabolite of $6 \mathrm{MM}$ was studied by TLC analysis after the fungi were fed with ${ }^{14} \mathrm{C}-6 \mathrm{MM}$. A spore suspension of $C$. globosum in $1 \mathrm{ml}$ medium was incubated with $1.0 \mathrm{mM}{ }^{14} \mathrm{C}-6 \mathrm{MM}$ for $12 \mathrm{hr}$, and the medium and resulting mycelia were separated by filtration through a $0.45 \mu \mathrm{m}$ millipore filter. The mycelia were transferred to $1 \mathrm{ml}$ of ethanol and the ${ }^{14} \mathrm{C}$-labelled compounds were extracted by boiling for $30 \mathrm{~min}$. The ${ }^{14} \mathrm{C}$-labelled compounds in the medium were also extracted by blending with $1 \mathrm{ml} n$-butanol. From these extracts, 100 $\mu \mathrm{l}$-aliquots were applied to a silica gel plate, and the plate was developed as mentioned above. The silica gel was scraped off in $1 \mathrm{~cm}$ widths, and the radioactivity of each region was measured. Bullera alba was incubated with $0.2 \mathrm{~mm}$ ${ }^{14} \mathrm{C}-6 \mathrm{MM}$ for $24 \mathrm{hr}$, and the experiment was carried out in the same manner.

\section{RESULTS}

\section{Effect of 6MM on germination of spores of C. globosum}

Under the present conditions, about $70 \%$ of the spores of $C$. globosum have emerged germ-tubes after $6 \mathrm{hr}$ of cultivation (Fig. 1). In the presence of $1.0 \mathrm{~mm}$ $6 \mathrm{MM}$, spore germination was almost completely prevented for the first $3 \mathrm{hr}$. The inhibitory effects were transient, however, and the phytoalexin simply lengthened the lag period before the germ-tube emission occurred. After cultivation 


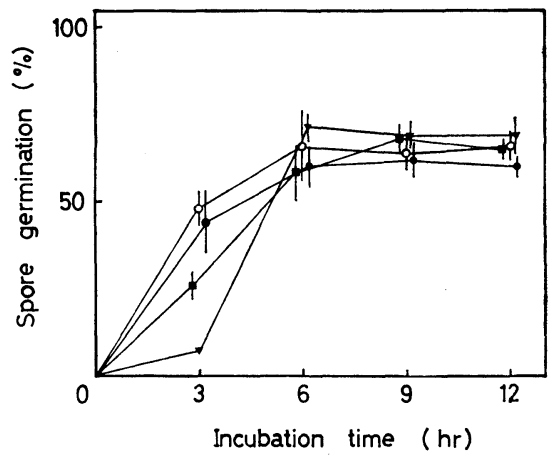

Fig. 1. Effect of $6 \mathrm{MM}$ on spore germination of C. globosum.

The symbols represent $0(\bigcirc), 0.1(\bullet), 0.5(\square)$ and $1.0(\nabla) \mathrm{mM} 6 \mathrm{MM}$. Points and bars are the means and standard deviations, respectively, obtained from five replicate experiments.

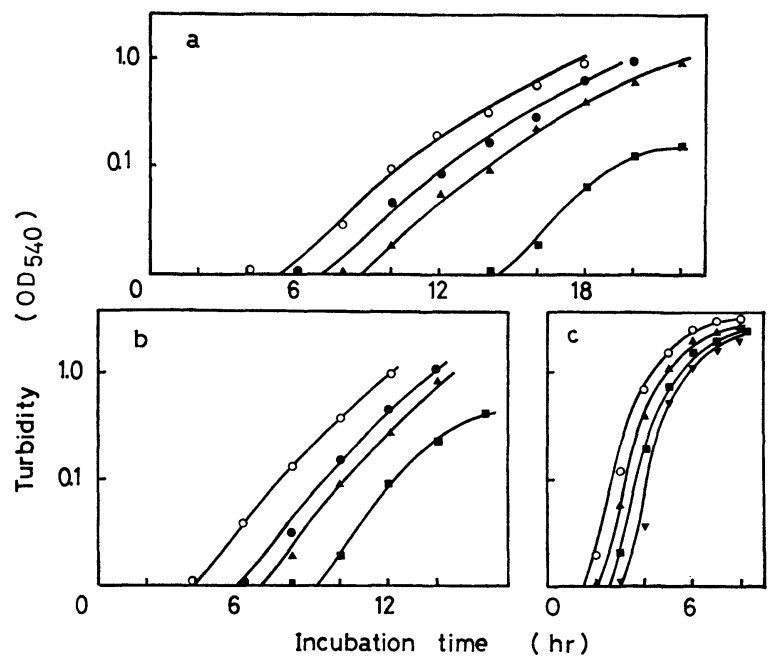

Fig. 2. Effect of 6MM on growth of B. alba (a), C. albicans (b) and S. aureus (c). The symbols represent $0(0), 0.1(\bullet), 0.2(\Delta), 0.5(\boldsymbol{\nabla})$ and $1.0(\nabla) \mathrm{mM} 6 \mathrm{MM}$. The growth of microorganisms was followed by turbidity measurements.

for $6 \mathrm{hr}$, the percentages of germination had reached a level similar to that of the control culture.

\section{Effect of 6MM on microbial growth}

Addition of $6 \mathrm{MM}$ to the cultures of yeasts and bacteria caused an extension of the lag period before active growth commenced (Fig. 2). The duration of $6 \mathrm{MM}$-induced growth inhibition was roughly proportional to the concentration of $6 \mathrm{MM}$ added to the culture. After the lag period, microbial growth resumed 


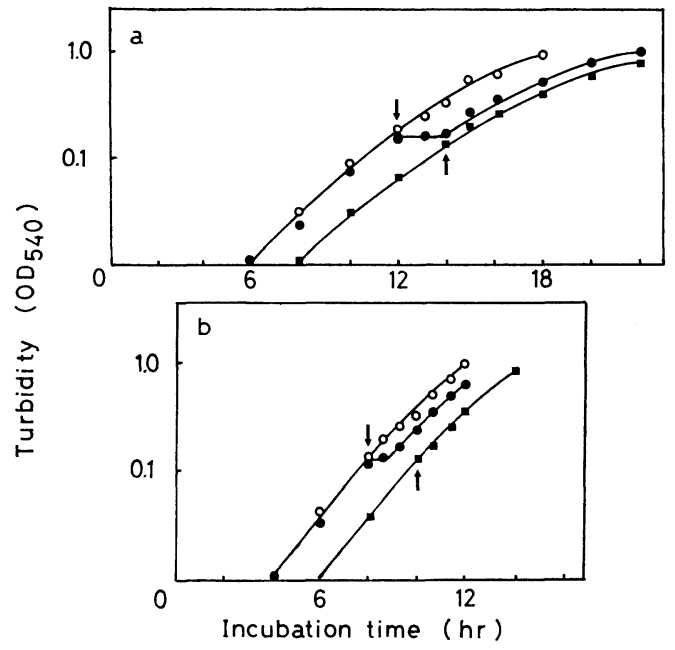

Fig. 3. Effect of successive additions of $0.2 \mathrm{~mm} 6 \mathrm{MM}$ on cultures of B. alba (a) and $C$. albicans (b).

The first addition of $0.2 \mathrm{~mm} 6 \mathrm{MM}$ was made at time zero ( $\boldsymbol{\square})$; upward arrows indicate the time of the second addition to the same culture. To one of the control cultures (๑), $0.2 \mathrm{mM} 6 \mathrm{MM}$ was added at the time indicated by downward arrows, and another culture $(O)$ received only ethanol at the same time.

at the same rate as in the control culture. In the culture of B. alba and C. albicans treated with $0.5 \mathrm{mM} 6 \mathrm{MM}$, however, the cell densities at the stationary phase were obviously lower than that in the absence of 6MM (Fig. 2a, b). Viable cells in these cultures, as determined by a colony count, was $40-50 \%$ of the control. These observations suggest that in these microorganisms, tolerance occurred after a transient period of sensitivity at a low concentration of $6 \mathrm{MM}$, but the phytoalexin appears to exert a fungicidal effect when applied at a higher concentration.

A further experiment was set up to test whether the microorganisms become tolerant to $6 \mathrm{MM}$ following exposure to it (Fig. 3). The yeasts were inoculated to the culture medium supplemented with $0.2 \mathrm{~mm} 6 \mathrm{MM}$. When the exponential growth resumed after the $6 \mathrm{MM}$-induced lag phase, $6 \mathrm{MM}$ was again added to the culture. Addition of the second does of $6 \mathrm{MM}(0.2 \mathrm{mM})$ did not show any discernible effect on the growth of the yeasts. On the other hand, $B$. alba and $C$. albicans which had not been previously treated with $6 \mathrm{MM}$ were sensitive and the addition of the phytoalexin resulted in an abrupt, but transient cessation of the increase in turbidity. It is likely that the tolerance of $B$. alba and C. albicans for $6 \mathrm{MM}$ was induced by the first dose of the phytoalexin.

The inhibitory effect of $6 \mathrm{MM}$ on $S$. aureus appeared to be influenced by the bacterial cell density. When an exponentially growing culture of $S$. aureus was inoculated at various cell density, the delay of the beginning of active growth was 


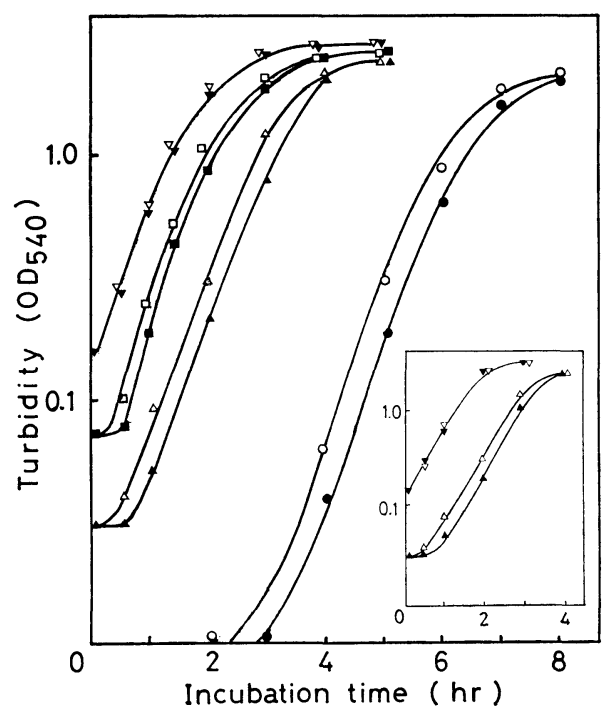

Fig. 4. Effect of $6 \mathrm{MM}$ on $S$. aureus at various bacterial cell densities. S. aureus culture in the exponential phase was inoculated into a $5 \mathrm{ml}$ medium containing $0.2 \mathrm{~mm}$ 6MM. Initial cell desity; $(\bullet), 0.2 \times 10^{4} ;(\Delta), 1.6 \times 10^{4} ;(\square), 1.0 \times 10^{5} ;(\nabla), 1.6 \times 10^{5} / \mathrm{ml}$. Open symbols represent respective control cultures without $6 \mathrm{MM}$. Insert figure shows the effect of $6 \mathrm{MM}$ on the culture of $S$. aureus pre-treated with $0.2 \mathrm{mM} 6 \mathrm{MM}$ for $12 \mathrm{hr}$.

inversely proportional to the number of bacteria in the culture (Fig. 4). The culture of $S$. aureus pre-treated with $0.2 \mathrm{~mm} 6 \mathrm{MM}$ was also tested in this series of experiments (Fig. 4). When the bacterial density was low, S. aureus showed a sensitivity to the second dose of $6 \mathrm{MM}$ similar to the first one. The tolerance of $S$. aureus, in contrast to $B$. alba and $C$. albicans, seemed to be caused in a manner other than an inducible factor.

\section{Loss of $6 M M$ from culture fluid}

The limited duration of growth inhibition caused by 6MM might be a consequence of enzymic degradation or transformation of the phytoalexin. To test this, changes in the $6 \mathrm{MM}$ concentration in the medium was followed during culture. When the culture medium was inoculated with the spores of C. globosum, the concentration of $6 \mathrm{MM}$ in the medium decreased gradually during the first 5-6 hr, and then rapidly after spore germination had been completed (Fig. 5a). In $B$. $a l b a, 6 \mathrm{MM}$ in the medium decreased after a short lag period, and reached a constant level of about $50 \%$ of the initial concentration (Fig. 5b). The level of $6 \mathrm{MM}$ in the media of $C$. albicans and $S$. aureus decreased only slightly (Fig. 5c, d). The restoration of growth of these microorganisms in a few hours after the addition of the phytoalexin implies that they acquired the tolerance to $6 \mathrm{MM}$ while the culture medium still contained enough phytoalexin to inhibit their growth. 


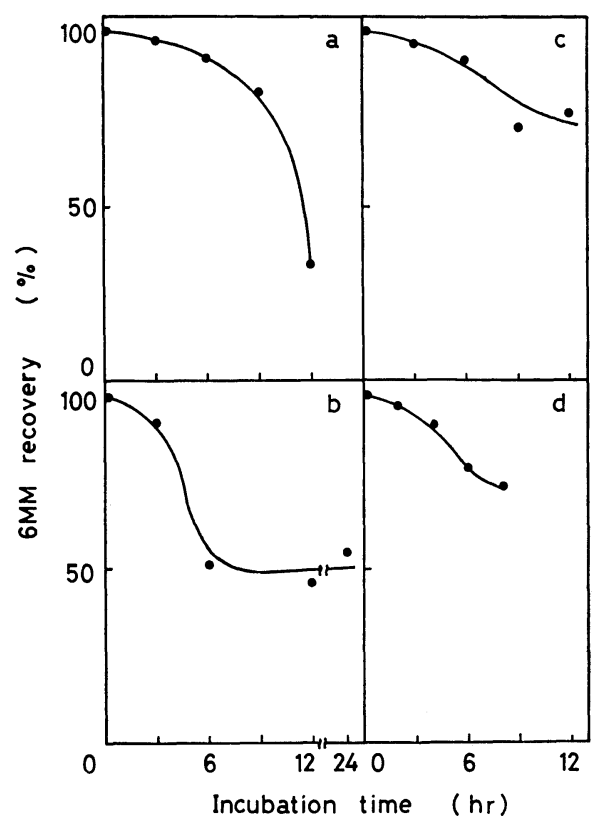

Fig. 5. Loss of 6MM from culture medium.

Cultures of $C$. globosum (a) and $S$. aureus (d) were supplemented with $1.0 \mathrm{~mm}$ ${ }^{14} \mathrm{C}-6 \mathrm{MM}$, and B. alba (b) and C. albicans (c) with $0.2 \mathrm{mM}{ }^{14} \mathrm{C}-6 \mathrm{MM}$. Aliquots of the culture $(0.1 \mathrm{ml})$ were removed at the time indicated and $6 \mathrm{MM}$ was extracted from the culture medium with $n$-butanol. The radioactivity of ${ }^{14} \mathrm{C}-6 \mathrm{MM}$ was measured after being separated by TLC. Data presented are the percentages of $6 \mathrm{MM}$ left to that added at time zero.

As the concentration of $6 \mathrm{MM}$ in the culture fluid of C. globosum and B. alba decreased appreciably during cultivation, possible metabolites of $6 \mathrm{MM}$ were investigated after incubation with these two microorganisms. In these experiments, $C$. globosum was cultured in the presence of ${ }^{14} \mathrm{C}-6 \mathrm{MM}$ and the extracts obtained from mycelia and medium were developed on a silica gel plate. About $70 \%$ of the $6 \mathrm{MM}$ added to the culture was recovered in the mycelia, and no ${ }^{14} \mathrm{C}$ labelled compound other than $6 \mathrm{MM}$ occurred in the extract from either the mycelia or culture fluid. This suggests that the mycelium of C. globosum posseses an ability to take up $6 \mathrm{MM}$ and deactivate it in a way other than degradation or transformation. The radioactivity in the cells was easily removed by washing with several portions of water or buffer $(40 \mathrm{~mm}$ Tris- $\mathrm{HCl}, \mathrm{pH} 7.2)$ indicating that the $6 \mathrm{MM}$ associated only loosely with the cellular components. The experiment on $B$. alba gave a result similar to that described for C. globosum. 


\section{DISCUSSION}

The results reported in this paper show that $6 \mathrm{MM}$ is a static antimicrobial compound at a low concentration. Pterocarpan phytoalexins have been shown to be static agents against fungi, yeasts and bacteria $(13,14)$. DENNY and VANETTEN (7), however, reported that medicarpin, an isoflavonoid phytoalexin from the jack bean, showed a fungicidal effect when applied at a high concentration. Spores treated with $90 \mu \mathrm{g}$ of the phytoalexin could not germinate even after being washed. The inhibitory effect of $6 \mathrm{MM}$ also depended on its concentration. Chaetomium globosum and $S$. aureus were transiently inhibited by $0.2 \mathrm{~mm} 6 \mathrm{MM}$ (Figs. 2 and 3). At a concentration greater than $0.5 \mathrm{~mm}$, however, the yields of these yeasts when the maximum growth was attained and their colony forming activities were obviously decreased compared to the control, suggesting that the phytoalexin exerts a fungicidal effect at a high concentration.

The microorganisms tested in this experiment expressed phytoalexin torelance after a period during which growth or germination was inhibited. $B$. alba and $C$. albicans pre-treated with $6 \mathrm{MM}$ were no longer sensitive to the phytoalexin when the second dose was added to the culture (Fig. 3). In these cells, the phytoalexin tolerance appears to be induced by the first dose of $6 \mathrm{MM}$. On the other hand, it is likely that $S$. aureus tolerates $6 \mathrm{MM}$ by a constitutive manner because the second dose of $6 \mathrm{MM}$ resulted in a growth inhibition similar to the first. WeINSTEIN et al. (15) reported that Erwinia carotovora detoxifies glycinol, one of the phytoalexins produced by soybeans, by a constitutive factor. By contrast, DenNy and VANETTEN (7) showed that an isolate of Nectria haematococca has the ability to adapt to a pterocarpan phytoalexin. These findings indicate that phytoalexins are tolerated by microorganisms in different ways.

The metabolism of phytoalexins has been studied by several workers (3-8), and cases have been reported in which microorganisms became insensitive to phytoalexin without loss of the compound from the culture medium $(7,9-11)$. Tolerance may occur in the following alternative ways: 1) $C$. globosum and $B$. alba incorporated an apprecialbe amount of $6 \mathrm{MM}$, but no degradation product or metabolite was detected in these cultures. This suggests that these microorganisms acquire phytoalexin tolerance by a mechanism without biochemical modification of the compound. 2) If $6 \mathrm{MM}$ blocks an essential metabolic step, cells could become tolerant by forming an alternate pathway. 3) Tolerance may also occur by a compartmentation of the toxic compound or binding it to a certain cellular component. Transferring a substance from cytoplasm to vacuoles, however, generally requires its transformation to a more hydrophilic compound (e.g. hydroxylation, glycosylation or conjugation with amino acid). The inducible nature of the tolerance mechanism in $B$. alba and C. albicans may favor the first hypothesis, though no direct evidence for this is available. However, that hypothesis can not explained the tolerance in $S$. aureus in which the duration 
of growth inhibition was dependent on the size of the bacterial inoculum. It is possible that the phytoalexin lost its toxicity by being adsorbed to cellular components or cell-surface structures when a sufficient number of attachment sites were present. Inactivation of some chemotherapeutic agents, such as sulfanylamide-derivatives and $\beta$-lactum antibiotics, by binding to serum protein has been recognized for many years $(16,17)$. A similar dependence of the inhibitory activity of phytoalexin on bacterial concentration has been reported by GNANAMANICKAN and PATIL (18) and WEINSTEIN et al. (15).

$6 \mathrm{MM}$ affected microbial growth in both the exponential and stationary phases. The site of action of $6 \mathrm{MM}$ is, on this account, presumed to be a process not characteristically altered according to the growth phase. The broad antimicrobial spectrum previously reported and the phase-independent effect of $6 \mathrm{MM}$ suggest that this phytoalexin interferes with a common process in the metabolism of these microorganisms.

The present experiments showed that many fungi are initially sensitive to $6 \mathrm{MM}$ but tolerance is rapidly developed in these microorganisms. If the phytoalexin plays a role as a defense substance, it should be accumulated at an infection site to a concentration sufficient for the complete inhibition of microbial growth. In some host-parasite systems, it was shown that phytoalexins accumulations in diseased plants exceeded the concentration required for the restriction of fungal growth. Cytological studies at the site of phytoalexin accumulation combined with in vitro experiments would help to solve the problem $(19,20)$.

This work was supported in part by a Grant-in-Aid from the Ministry of Education, Science and Culture of Japan.

\section{REFERENCES}

I) J. Kuć, In Microbial Toxins VIII, ed. by K. Solomon, Academic Press, New York (1972), p. 211.

2) H. Grisebach and J. Ebel, Angw. Chem. Int. Ed. Engl., 17, 635 (1978).

3) van den J. Heuvel and H. D. VanEtten, Physiol. Plant Pathol., 3, 327 (1973).

4) R. S. Burden, J. A. Bailey and G. G. Vincent, Phytochemistry, 13, 1789 (1974).

5) van den J. Heuvel, H. D. Vanetten, J. W. Serum, D. L. Coffen and T. H. Williams, Phytochemistry, 13, 1129 (1974).

6) H. D. Vanetten, S. G. Pueppke and T. L. Kelsey, Phytochemistry, 14, 1103 (1975).

7) T. P. Denny and H. D. VanEtten, Physiol. Plant Pathol., 19, 419 (1981).

8) T. P. Denny and H. D. VanEtten, Phytochemistry, 21, 1023 (1982).

9) van den J. Heuvel and J. A. Glazener, Neth. J. Plant Pathol., 81, 125 (1975).

10) M. Yoshikawa, K. Yamauchi and H. Masago, Physiol. Plant Pathol., 14, 157 (1979).

11) T. P. Denny and H. D. VanEtten, Phytopathology, 71, 213 (1981).

12) F. Kurosaki and A. Nishi, Phytochemistry, 22, 669 (1983).

13) I. A. M. Cruickshank and D. R. Perrin, Aust. J. Biol. Sci., 14, 336 (1961).

14) N. T. Keen and B. W. Kennedy, Physiol. Plant Pathol., 4, 173 (1974).

15) L. I. Weinstein, M. G. Hahn and P. Albersheim, Plant Physiol., 68, 358 (1981). 
16) G. ZBinden, In Molecular Modification in Drug Design, ed. by R. F. Gold, American Chemical Society, Washington, D. C. (1964), p. 25.

17) M. C. Meyer and D. E. Guttman, J. Pharm. Sci., 57, 895 (1968).

18) S. S. Gnanamanickan and S. S. Patil, Physiol. Plant Pathol., 10, 159 (1977).

19) M. Yoshikawa, K. Yamauchi and H. Masago, Physiol. Plant Pathol., 12, 73 (1978).

20) J. A. Bailey and B. J. Deverall, Physiol. Plant Pathol., 1, 435 (1971). 